
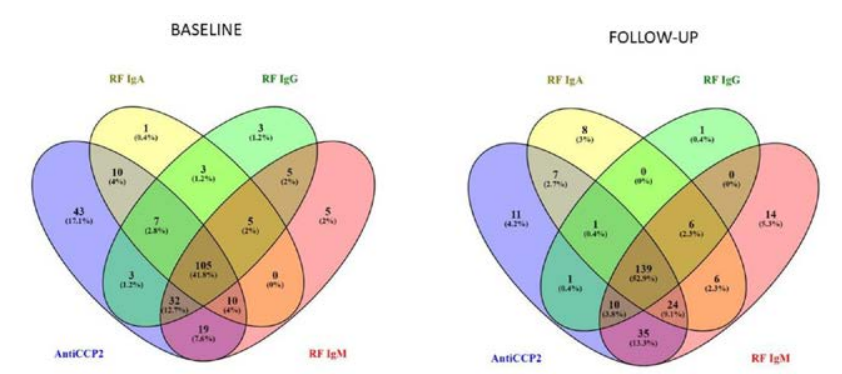

Figure 1. Comparison of serum autoantibody profile in rheumatoid arthritis patients during baseline enrolment and 10 years follow-up.

Acknowledgements: The authors would like to thank the Director General of Health, Ministry of Health Malaysia for supporting this study. The authors are also indebted to participants for their kind participation. This study was financially supported by the Ministry of Health, Malaysia (JPP-IMR 08-012; 18-051).

Disclosure of Interests: None declared

DOI: 10.1136/annrheumdis-2021-eular.1714

\section{POS0458 IDENTIFICATION OF HUB GENES AND MOLECULAR PATHWAYS IN PATIENTS WITH RHEUMATOID ARTHRITIS BY BIOINFORMATICS ANALYSIS}

L. Cheng ${ }^{1}$, S. X. Zhang ${ }^{2,3,4}$, S. Song ${ }^{2,3,4}$, C. Zheng ${ }^{1}$, X. Sun ${ }^{1}$, S. Feng ${ }^{1}$, T. Kong ${ }^{1}$, G. Shi ${ }^{5}$, X. Li ${ }^{2,3}$, P. F. He ${ }^{6}$, Q. Yu ${ }^{1,6} .{ }^{1}$ Shanxi Medical University, School of Management, Taiyuan, China; ${ }^{2}$ Shanxi Medical University, Department of Rheumatology, Taiyuan, China; ${ }^{3}$ Shanxi Li Xiaofeng Medical Groups, Department of Rheumatology, Taiyuan, China; ${ }^{4}$ Ministry of Education, Key laboratory of Cellular Physiology at Shanxi Medical University, Taiyuan, China; ${ }^{5}$ Shanxi Medical University, Basic Medical College, Taiyuan, China; ${ }^{6}$ Shanxi Medical University, Institute of Medical Data Sciences, Taiyuan, China

Background: Rheumatoid arthritis (RA) is a chronic, inflammatory synovitis based systemic disease of unknown etiology ${ }^{1}$. The genes and pathways in the inflamed synovium of RA patients are poorly understood.

Objectives: This study aims to identify differentially expressed genes (DEGs) associated with the progression of synovitis in RA using bioinformatics analysis and explore its pathogenesis ${ }^{2}$.

Methods: RA expression profile microarray data GSE89408 were acquired from the public gene chip database (GEO), including 152 synovial tissue samples from RA and 28 healthy synovial tissue samples. The DEGs of RA synovial tissues were screened by adopting the R software. The Gene Ontology (GO) and Kyoto Encyclopedia of Genes and Genomes (KEGG) pathway enrichment analysis were performed. Protein-protein interaction (PPI) networks were assembled with Cytoscape software.

Results: A total of 654 DEGs (268 up-regulated genes and 386 down-regulated genes) were obtained by the differential analysis. The GO enrichment results showed that the up-regulated genes were significantly enriched in the biological processes of myeloid leukocyte activation, cellular response to interferon-gamma and immune response-regulating signaling pathway, and the down-regulated genes were significantly enriched in the biological processes of extracellular matrix, retinoid metabolic process and regulation of lipid metabolic process. The KEGG annotation showed the up-regulated genes mainly participated in the staphylococcus aureus infection, chemokine signaling pathway, lysosome signaling pathway and the down-regulated genes mainly participated in the PPAR signaling pathway, AMPK signaling pathway, ECM-receptor interaction and so on. The 9 hub genes (PTPRC, TLR2, tyrobp, CTSS, CCL2, CCR5, B2M, fcgr1a and PPBP) were obtained based on the String database model by using the Cytoscape software and cytoHubba plugin ${ }^{3}$.

Conclusion: The findings identified the molecular mechanisms and the key hub genes of pathogenesis and progression of RA.

\section{REFERENCES:}

[1] Xiong Y, Mi BB, Liu MF, et al. Bioinformatics Analysis and Identification of Genes and Molecular Pathways Involved in Synovial Inflammation in Rheumatoid Arthritis. Med Sci Monit 2019;25:2246-56. doi: 10.12659/MSM.915451 [published Online First: 2019/03/28]

[2] Mun S, Lee J, Park A, et al. Proteomics Approach for the Discovery of Rheumatoid Arthritis Biomarkers Using Mass Spectrometry. Int J Mol Sci 2019;20(18) doi: 10.3390/ijms20184368 [published Online First: 2019/09/08]

[3] Zhu N, Hou J, Wu Y, et al. Identification of key genes in rheumatoid arthritis and osteoarthritis based on bioinformatics analysis. Medicine (Baltimore) 2018;97(22):e10997. doi: 10.1097/MD.0000000000010997 [published Online First: 2018/06/01]
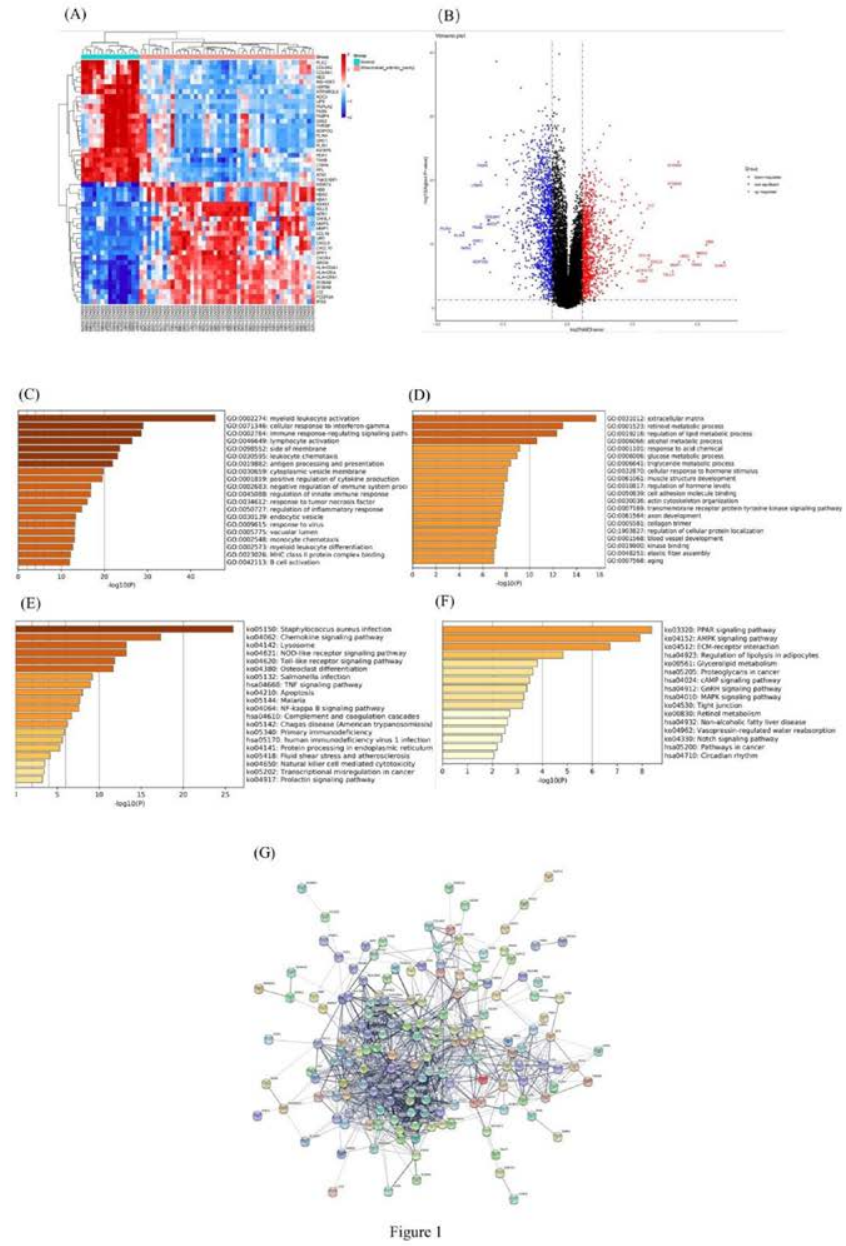

(A)Heat map of the top 50 up-regulated differentially expressed genes: Heatmaps of the differentially expressed genes (DEGs) including the 25 most upregulated and 25 most down-regulated genes. (B) Volcano map of gene expression: The horizontal axis is $\log 2 \mathrm{FC}$ and the vertical axis is $\log 10$ adj.p.val. Each dot represents a gene, blue represents down-regulated gene, red represents up-regulated gene, and black represents an in differentially expressed gene. (C,D)Visualization GO enrichment analysis: Go analysis showed that the function of up-regulated genes was mainly reflected in 20 aspects, and $\mathrm{B}$ cell activation had the lowest $\mathrm{p}$ value. The function of down regulated genes is mainly reflected in 20 aspects, and aging is the lowest in P value. (E,F)KEGG pathway enrichment analysis: Among the signaling pathways of up-regulated genes, the largest P value was Staphylococcus aureus. PPAR signaling pathway has the highest $p$ value among the signaling pathways of down regulated genes. (G)Protein-protein interaction network of differentially expressed genes: The 9 hub genes were selected,
they were PTPRC, TLR2, tyrobp, CTSS, CCL2, CCR5, B2M, FCGR1A and PPBP.

Acknowledgements: This project was supported by National Science Foundation of China (82001740), Open Fund from the Key Laboratory of Cellular Physiology (Shanxi Medical University) (KLCP2019) and Innovation Plan for Postgraduate Education in Shanxi Province (2020BY078).

Disclosure of Interests: None declared

DOI: 10.1136/annrheumdis-2021-eular.1938

\section{POS0459 \\ UNSUPERVISED CLUSTERING IDENTIFIES UNIQUE SUBSETS OF PATIENTS IN A RACIALLY AND ETHNICALLY DIVERSE RHEUMATOID ARTHRITIS COHORT}

G. Lui ${ }^{1}$, E. Noss ${ }^{2}$, N. Singh ${ }^{2}$, J. Andrews ${ }^{2}$, J. Graf ${ }^{3}$, K. Wysham ${ }^{2,4} \cdot{ }^{1}$ University of Washington, Bioengineering, Seattle, United States of America; ${ }^{2}$ University of Washington, Rheumatology/Medicine, Seattle, United States of America; ${ }^{3}$ University of California San Francisco Parnassus Campus, Rheumatology/ Medicine, San Francisco, United States of America; ${ }^{4}$ VA Puget Sound Health Care System, Rheumatology/Medicine, Seattle, United States of America

Background: Single biomarkers have limited utility to date in guiding RA clinical care. Machine learning algorithms may better identify and stratify RA patients with differential outcomes.

Objectives: To determine if unsupervised machine learning methods can be employed in a racially and ethnically diverse RA cohort to identify clusters of patients with different disease activity trajectories, as measured by DAS28ESR. Methods: Data are derived from the longitudinal, observational University of California, San Francisco RA Cohort. Along with routine labs, medications and disease activity assessments, a multiple biomarker of disease activity (MBDA) 
Table 1. Demographics and clinical characteristics of the RA cohort. Biomarkers significantly associated with DAS28ESR were determined by Lasso regression Values listed are per standard deviation of each biomarker.

\begin{tabular}{|c|c|c|c|c|c|c|}
\hline & & Overall $(\mathrm{N}=373)$ & Cluster $1(\mathrm{~N}=116)$ & Cluster $2(\mathrm{~N}=70)$ & Cluster $3(\mathrm{~N}=14)$ & $\begin{array}{l}\text { Cluster } 4 \\
(\mathrm{~N}=173)\end{array}$ \\
\hline \multirow[t]{3}{*}{ Demographics: } & $\begin{array}{l}\text { Age } \\
\text { Eemale Sex } \\
\text { Race: Hispanic/Latino } \\
\text { Asian }\end{array}$ & $\begin{array}{l}54.83 .6 \\
3188(85 \%) \\
181 \text { (49\%) } \\
123(33 \%)\end{array}$ & $\begin{array}{l}63.6 \pm 9.7 \\
101(87 \%) \\
22(19 \%) \\
73(63 \%)\end{array}$ & $\begin{array}{l}50.8 \pm 14.9 \\
57(81 \%) \\
4767 \%) \\
8(11 \%)\end{array}$ & $\begin{array}{c}58.2 \pm 15.8 \\
11(79 \%) \\
56(36 \%) \\
6(43 \%)\end{array}$ & $\begin{array}{l}50.3+12,1 \\
149(86 \%) \\
107(62 \%) \\
36(21 \%)\end{array}$ \\
\hline & Black & $35(9 \%)$ & $12(10 \%)$ & $8(11 \%)$ & $2(14 \%)$ & $13(8 \%)$ \\
\hline & White \& Other & 34 (9\%) & $9(7 \%)$ & 7 (10\%) & $1(7 \%)$ & $17(10 \%)$ \\
\hline \multirow[t]{6}{*}{ Clinical Characteristics: } & $\begin{array}{l}\text { Rheymatoid Factor } \\
\text { ACPA } \\
\text { Disease Duration }\end{array}$ & $\begin{array}{l}315(85 \%) \\
297(80 \%) \\
7.8 \pm 7.6)\end{array}$ & $\begin{array}{l}104(90 \%) \\
98(85 \%) \\
13.7 \pm 9.7\end{array}$ & $\begin{array}{l}56(80 \%) \\
54(77 \%) \\
5.4 \pm 4.6\end{array}$ & $\begin{array}{l}13(93 \%) \\
12(86 \%) \\
6.7 \pm 5.7\end{array}$ & $\begin{array}{r}142(82 \%) \\
133(77 \%) \\
4.9 \pm 3.8\end{array}$ \\
\hline & CsDMARD & $344(92 \%)$ & $108(93 \%)$ & $63(90 \%)$ & $13(93 \%)$ & $160(93 \%)$ \\
\hline & Biologic DMARD & $185(50 \%)$ & 45 (39\%) & $30(43 \%)$ & $4(29 \%)$ & $106(61 \%)$ \\
\hline & Prednisone Dose & $6.7 \pm 3.8$ & $6.0 \pm 4.0$ & $8.6 \pm 4.9$ & $5.8 \pm 1.4$ & $6.3 \pm 2.8$ \\
\hline & Body Mass Index & $28.2 \pm 4.5$ & $26.6 \pm 3.8$ & $28.7 \pm 3.8$ & $28.0 \pm 6.2$ & $29.1 \pm 4.7$ \\
\hline & DAS28ESR & $4.2 \pm 1.1$ & $4.2 \pm 1.0$ & $5.5 \pm 0.8$ & $3.9 \pm 0.8$ & $3.7 \pm 0.9$ \\
\hline \multirow[t]{5}{*}{ Lasso Results: } & EGF & $-0.16^{\star}$ & $-0.41^{\star \star \star}$ & -- & -- & $-0.20^{\star \star}$ \\
\hline & Leptin & $0.15^{\star *}$ & -- & $0.21^{*}$ & -- & $0.21^{* *}$ \\
\hline & c-reactive protein & $0.34^{\star *}$ & $0.51^{* \star *}$ & -- & -- & -- \\
\hline & VCAM1 & -- & -- & -- & $-0.73^{*}$ & -- \\
\hline & YKL40 & -- & $0.26^{*}$ & -- & -- & -- \\
\hline
\end{tabular}

-EGF: epidermal growth factor; VCAM1: Vascular cell adhesion protein 1; YKL40: Chitinase-3-like protein 1.- ${ }^{*} \mathrm{p}<0.05,{ }^{* *} \mathrm{p}<0.01,{ }^{* * *} \mathrm{p}<0.001$

panel was obtained at each visit. The MBDA measures 12 serum biomarkers. Four patient clusters were identified by unsupervised K-prototype clustering after collapsing all observations into a cross sectional dataset. Plots were created to display longitudinal disease activity trajectories for each cluster. Lasso regression was applied to identify biomarkers associated with DAS28ESR.

Results: We identified 4 distinct clusters in our cohort (Table 1) with visually different disease activity trajectories (Fig. 1). Cluster $1(n=116)$ was older $(63.6 \pm 9.7)$, had the highest proportion of Asian participants $(n=73,63 \%)$ with the most study visits and longest disease duration. Cluster $2(n=70)$ had the highest mean DAS28ESR $(5.5 \pm 0.7)$, and the highest mean dose of prednisone $(8.6 \pm 4.9 \mathrm{mg} / \mathrm{day})$. Cluster 3 had the lowest number of participants ( $n=14)$, study visits and lowest biologic use (28.6\%). Cluster 4 was the largest cluster $(n=173)$ with the shortest disease duration $(4.9 \pm 3.8$ years) and highest biologic use (61.3\%). In the Lasso regressions, leptin was found to have significant positive associations with DAS28ESR in the whole group as well as Clusters 2 and 4. EGF had negative associations with DAS28ESR in the whole group, Cluster 1 and 4. CRP had positive associations with DAS28ESR in the whole group and Cluster 1. YKL40 and VCAM1 were found to have significant associations in Clusters 1 and 3, respectively. Conclusion: We identified 4 unique clusters of RA patients in a racially and ethnically diverse longitudinal cohort with different disease activity trajectories and biomarkers associated with disease activity. Although additional work is needed to explore longitudinal outcomes in each cluster, the application of machine learning methods may identify unique combinations of patient and disease characteristics influencing RA clinical outcomes.

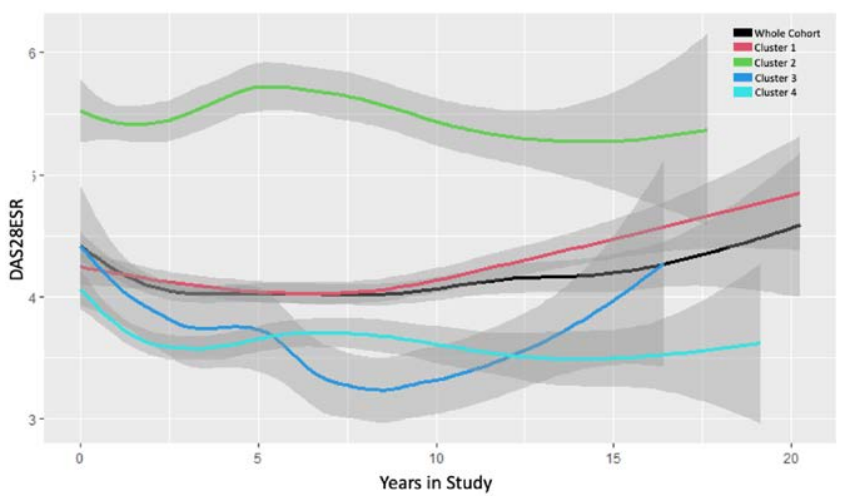

Figure 1. DAS28ESR trajectory plots with $95 \% \mathrm{Cls}$ for the whole cohort and by cluster.

Acknowledgements: This work was supported by the Rheumatology Research Foundation Scientist Development Award.

Disclosure of Interests: None declared

DOI: 10.1136/annrheumdis-2021-eular.1946

\begin{tabular}{|l|l}
\hline POS0460 & ASSOCIATION OF ANTI-CITRULLINATED \\
PROTEIN ANTIBODIES OF IGA SUBCLASSES \\
WITH SUSTAINED REMISSION AND FLARE IN \\
RHEUMATOID ARTHRITIS
\end{tabular}

M. V. Sokolova ${ }^{1,2}$, J. Rech ${ }^{1,2}$, M. Hagen ${ }^{1,2}$, G. Schett ${ }^{1,2}$, U. Steffen (née Harre) ${ }^{1,2}$. ${ }^{1}$ Friedrich-Alexander-University Erlangen-Nuremberg and Universitätsklinikum Erlangen, Department of Internal Medicine 3 - Rheumatology and Immunology, Erlangen,
Germany; ${ }^{2}$ Friedrich-Alexander-University Erlangen-Nuremberg and Universitätsklinikum Erlangen, Deutsches Zentrum für Immuntherapie, Erlangen, Germany

Background: Understanding key mechanisms of flare development and sustained remission is one of the acute goals in modern rheumatology. Anti-citrullinated protein antibodies (ACPA) are the most abundant and specific autoantibodies in rheumatoid arthritis (RA) patients. However, the impact of ACPA of IgA isotype is poorly defined. IgA ACPA were previously shown to have a higher percentage of IgA2 in comparison to total IgA; and a correlation between IgA2\% ACPA with the DAS28 score was observed in a previous study [1]. Of note, IgA1 and IgA2 were shown to exhibit different effector functions, with IgA2 being pro-inflammatory, which might be the background for its role in RA [1].

Objectives: We aimed to investigate, whether IgA ACPA could be used as a predictive factor for flare development in RA; and to look further into the changes in IgA ACPA levels in patients remaining in stable remission versus patients developing flare. Methods: We analysed serum of 111 patients from a multicentre randomized controlled trial 'RETRO'. The study observational period was 12 months. Patients in the trial had to be in stable remission (DAS28-ESR $<2.6$ ) for a minimum of 6 months and were randomized into 3 different treatment arms: continuation of treatment, tapering by $50 \%$ or a gradual tapering until discontinuation [2]. IgA ACPA concentrations were measured with an enzyme-linked immunosorbent assay on CCP2-pre-coated plates. Results: $60 \%$ of patients had IgG-ACPA. IgA ACPA levels were higher among the IgG-ACPA-positive patients (median 4.7 versus $2.24 \mu \mathrm{g} / \mathrm{ml}, \mathrm{p}<0.0001$ ) Baseline IgA1 and 2 ACPA levels were not different between patients who had a flare later on in the study period and those remaining in remission, showing no predictive value for flare development. However, the percentage of IgA2 in ACPA was correlating with the first registered DAS28 after flare $(r=0.36, p=0.046)$. After the 12 months study period, IgA2 ACPA as well as $\lg A 2 \%$ ACPA decreased significantly in patients who remained in stable remission by $17.5 \%$ (median, $p<0.0001)$ and $13.6 \%(p=0.0006)$, respectively. By contrast, there was no significant change in IgA2 ACPA levels over time in patients who developed a flare. IgA1 ACPA levels remained stable over time. Disease management strategies did not seem to influence IgA ACPA levels in a specific way, as baseline levels were similar between patients on biological and conventional DMARDs and changes in levels after 12 months did not depend on the assignment to either of the study arms.

Conclusion: Neither IgA1 nor IgA2 ACPA levels were predictive of flare development or associated with treatment strategies (though rituximab, JAK-inhibitors and abatacept were not amongst treatment options). However, in patients remaining in sustained remission after 1 year a decrease in IgA2 and IgA2\% ACPA was observed and IgA2\% ACPA was associated with DAS28 score registered after flare. This could be an indication towards ACPA of IgA2 isotype contributing to the severity of flare, alongside other factors, and its reduction being associated with a prolonged state of remission.

\section{REFERENCES:}

[1] Steffen U, Koeleman CA, Sokolova MV, et al. IgA subclasses have different effector functions associated with distinct glycosylation profiles. Nat Commun 11, 120 (2020).

[2] Haschka J, Englbrecht M, Hueber AJ, et al. Relapse rates in patients with rheumatoid arthritis in stable remission tapering or stopping antirheumatic therapy: interim results from the prospective randomised controlled RETRO study. Ann Rheum Dis. 75:45-51 (2016)

Disclosure of Interests: None declared

DOI: 10.1136/annrheumdis-2021-eular.2004 